\title{
PROTOCOLO DE TESTES DE ACEITAÇÃO EM EQUIPAMENTOS DE IMAGEM POR RESSONÂNCIA MAGNÉTICA*
}

\author{
Alessandro André Mazzola ${ }^{1}$, Silvio Bruni Herdade ${ }^{2}$, Hilton Augusto $\mathrm{Koch}^{3}$, Antonio Carlos \\ Pires Carvalho ${ }^{4}$
}

\begin{abstract}
Resumo OBJETIVO: Este trabalho tem como objetivo criar um protocolo de testes de aceitação para equipamentos de imagem por ressonância magnética e demonstrar como e quais tipos de dispositivos de teste podem ser usados para a coleta de dados. MATERIAIS E MÉTODOS: Para cada um dos 15 testes selecionados foram elaborados a definição, o procedimento, a forma de análise e o critério de aceitação. RESULTADOS: Através dos testes de aceitação descritos é possível verificar características técnicas que constam nas propostas de venda dos fabricantes, assim como estabelecer valores de referências para serem utilizados em posteriores testes de constância. CONCLUSÃO: Futuros programas de garantia da qualidade em imagem por ressonância magnética devem considerar testes semelhantes ou iguais aos descritos neste trabalho. Unitermos: Imagem por ressonância magnética; Controle de qualidade.
\end{abstract}

Abstract Acceptance testing protocol for magnetic resonance imaging systems.

OBJECTIVE: To develop an acceptance testing protocol for magnetic resonance imaging systems and to show which and how phantoms could be used to collect data. MATERIALS AND METHODS: The definition, procedure, analysis and the acceptation criteria were created for each of 15 selected tests. RESULTS: These tests allow the evaluation of technical specifications stated on the manufacturer's sales proposal and to establish reference values to be used in subsequent constancy tests. CONCLUSION: Future quality assurance programs in magnetic resonance imaging should consider employing tests similar or equal to the ones described in this paper.

Key words: Magnetic resonance imaging; Quality control.

\section{INTRODUÇÃO}

Uma grande quantidade de equipamentos de imagem por ressonância magnética (IRM) vem sendo instalada no Brasil, principalmente nos últimos cinco anos. Segundo dados do Instituto Brasileiro de Geografia e Estatística (IBGE) ${ }^{(\mathbf{1})}$, em 1999 havia 289 equipamentos de ressonância magnética (RM) instalados no Brasil, sendo a região Sudeste a detentora da maior quantidade (176), seguida pelas regiões Sul (42) e Nordeste (42), Centro-Oeste (23) e Norte (6). Estima-se que estes números cresce-

* Trabalho realizado no Hospital Moinhos de Vento, Porto Alegre, RS.

1. Aluno do Programa de Pós-graduação (Mestrado) em Radiologia da Faculdade de Medicina da Universidade Federal do Rio de Janeiro (UFRJ).

2. Professor Associado do Instituto de Física da Universidade de São Paulo (USP).

3. Professor Titular do Departamento de Radiologia da Faculdade de Medicina da UFRJ.

4. Professor Adjunto do Departamento de Radiologia da Faculdade de Medicina da UFRJ, Coordenador Adjunto do Programa de Pós-graduação em Radiologia da UFRJ.

Endereço para correspondência: Dr. Alessandro André Mazzola. Rua Paulino Chaves, 123/301. Porto Alegre, RS, 90640200. E-mail: aam.ez@terra.com.br

Recebido para publicação em 22/10/2003. Aceito, após revisão, em 2/8/2004. ram cerca de $30 \%$ nos últimos três anos, o que representa a instalação de aproximadamente 86 novos equipamentos.

Uma série de condições referentes ao funcionamento do equipamento e de seus acessórios deve ser observada, tais como, intensidade do campo magnético principal, homogeneidade do campo magnético, amplitude e tempo de subida dos gradientes, razão sinal-ruído e uniformidade de sinal das bobinas, para que se possa obter imagens com qualidade e tempo de exame adequados para o diagnóstico.

A normatização e recomendação sobre testes de aceitação em equipamentos de IRM se restringe a trabalhos feitos pela Associação Americana de Físicos em Medicina (AAPM) ${ }^{(\mathbf{1 - 8})}$, Associação dos Fabricantes de Equipamentos dos Estados Unidos (NEMA) ${ }^{(\mathbf{9 - 1 6})}$, Colégio Americano de Radiologia $(\mathrm{ACR})^{(\mathbf{1 7 )}}$ e Instituto de Físicos em Medicina do Reino Unido (IPEM) ${ }^{(\mathbf{1 8})}$. Não existe nenhuma publicação, recomendação ou normatização nacional sobre testes de aceitação ou controle de qualidade para esta técnica de diagnóstico por imagem. O ACR, em especial, criou o progra- ma de Acreditação em Imagem por Ressonância Magnética, baseado nas exigências contidas em sua publicação e nas publicações da AAPM.

Este trabalho tem como objetivo criar um protocolo de testes de aceitação para equipamentos de IRM e demonstrar como e quais tipos de dispositivos de teste podem ser usados para a coleta de dados.

\section{MATERIAIS E MÉTODOS}

Para cada teste foi elaborada uma definição, o procedimento, a forma de análise e o critério de aceitação. O critério de aceitação foi especificado em termos de limites de ação que definem a faixa de valores aceitáveis, fora dos quais ações corretivas são necessárias.

Com a finalidade de verificar a forma de análise e aplicabilidade, todos os testes foram realizados em dois equipamentos de IRM de 1,5 tesla que possuem magneto supercondutor, de um hospital privado de grande porte.

Testes que necessitavam de licença ou equipamento específico, fornecido pelo 
fabricante somente para os seus engenheiros de campo, não fizeram parte deste protocolo.

Para a realização dos testes de aceitação podem ser utilizados dispositivos comerciais e dispositivos do próprio fabricante do equipamento. Foram utilizados, neste estudo, os dispositivos de teste UAL ("uniformity and linearity") (Nuclear Associates, Inc., Hicksville, NY) (19), 3DRAS (“3D resolution and slice") (Nuclear Associates, Inc., Hicksville, NY) ${ }^{(\mathbf{1 9 )}}$, conforme mostra a Figura 1, e dispositivos do próprio fabricante dos equipamentos da instituição à qual um dos autores pertence. Podem ser utilizados outros tipos de dispositivos de teste e também construídos conforme sugerido pela literatura ${ }^{(\mathbf{1 , 3 - 8 , 1 8})}$.

O Quadro 1 mostra a relação dos dispositivos utilizados e o teste associado.

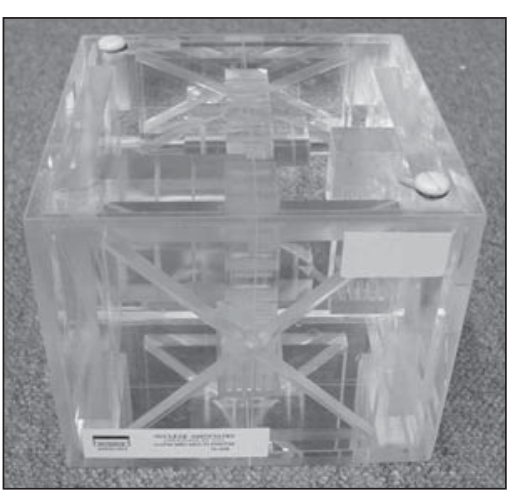

A

Figura 1. A: Dispositivo de teste 3DRAS. B: Dispositivo de teste UAL.

Quadro 1 Relação dos dispositivos e o respectivo teste em que pode ser utilizado.

\begin{tabular}{|c|c|}
\hline Dispositivo de teste & Teste que realiza \\
\hline 3DRAS (3D "resolution and slice") & $\begin{array}{l}\text { Exatidão da espessura de corte } \\
\text { Exatidão do posicionamento e espaçamento de corte } \\
\text { Resolução espacial de alto contraste }\end{array}$ \\
\hline UAL (“Uniformity and linearity”) & $\begin{array}{l}\text { Uniformidade da imagem } \\
\text { Distorção geométrica ou linearidade espacial } \\
\text { Análise de artefatos na imagem }\end{array}$ \\
\hline RSR (razão sinal-ruído) crânio & $\begin{array}{l}\text { Razão sinal-ruído } \\
\text { Uniformidade } \\
\text { Freqüência central } \\
\text { Ganho de transmissão } \\
\text { Checagem das bobinas de radiofreqüência } \\
\text { Interferência de radiofreqüência entre os cortes }\end{array}$ \\
\hline RSR corpo & $\begin{array}{l}\text { Razão sinal-ruído } \\
\text { Uniformidade }\end{array}$ \\
\hline Esfera de $100 \mathrm{~mm}$ & $\begin{array}{l}\text { Razão sinal-ruído } \\
\text { Uniformidade }\end{array}$ \\
\hline Cilindro de desacoplamento & $\begin{array}{l}\text { Razão sinal-ruído } \\
\text { Uniformidade }\end{array}$ \\
\hline Arranjo pélvico & $\begin{array}{l}\text { Razão sinal-ruído } \\
\text { Uniformidade }\end{array}$ \\
\hline Arranjo CTL (cervical-torácico-lombar) & $\begin{array}{l}\text { Razão sinal-ruído } \\
\text { Uniformidade }\end{array}$ \\
\hline Punho & $\begin{array}{l}\text { Razão sinal-ruído } \\
\text { Uniformidade }\end{array}$ \\
\hline Braino MRS (espectroscopia por RM) & Verificação da espectroscopia \\
\hline
\end{tabular}

\section{RESULTADOS}

Um total de 15 testes foi selecionado, conforme mostra o Quadro 2.

Para todos os testes que necessitam obtenção de imagens dos dispositivos, devem ser adquiridas imagens spin eco com ponderação em T1, com espessura de corte e intervalo compatíveis com os protocolos clínicos utilizados na rotina do serviço ou especificados de acordo com o tipo de teste.

A descrição dos testes foi elaborada sob a forma de um protocolo, para facilitar sua execução e entendimento.

Quadro 2 Testes de aceitação selecionados.

\begin{tabular}{|l|}
\hline Teste de aceitação \\
\hline 1. Observação física \\
2. Verificação da blindagem de radiofreqüên- \\
cia (RF) \\
3. Consumo do criogênio \\
4. Homogeneidade do campo magnético prin- \\
cipal (Bo $)$ \\
5. Exatidão da espessura de corte \\
6. Exatidão do posicionamento e do espaça- \\
mento de corte \\
7. Resolução espacial de alto contraste \\
8. Uniformidade da imagem \\
9. Linearidade espacial ou distorção geomé- \\
trica \\
10. Análise de artefatos na imagem \\
11. Estabilidade do campo magnético ou fre- \\
qüência central \\
12. Ganho de transmissão ou atenuação \\
13. Checagem das bobinas de RF \\
14. Interferência de RF entre os cortes \\
15. Verificação da espectroscopia
\end{tabular}

\section{Teste 1 - Observação física Definição}

A observação física envolve uma vistoria de todos os itens contidos na proposta de venda e especificados pelo fabricante.

\section{Procedimento e análise}

Fazer um inventário para determinar todos os itens identificados na proposta de venda. Determinar se equipamentos como sistema de impressão, intercomunicação com paciente, botões de alarme e movimentação da mesa do paciente estão funcionando corretamente. Uma inspeção de itens relacionados à segurança deve ser avaliada $^{(6,8)}$. 


\section{Critério de aceitação}

Qualquer item fora de especificação, com defeito, ou faltando, deve ser comunicado por escrito ao engenheiro responsável pela instalação do equipamento, assim como para a direção da instituição.

\section{Teste 2 - Verificação da blindagem de radiofreqüência (RF)}

Definição

A blindagem de RF serve para garantir que existe um isolamento eletromagnético do ambiente externo à sala de exames. A blindagem é geralmente testada por uma empresa independente ou pelo próprio fabricante do equipamento. Este teste é realizado colocando-se uma antena do lado externo e transmitindo sinais de várias freqüências através da blindagem. A atenuação do sinal é determinada por comparação com valores de referência obtidos em pontos onde não há blindagem ${ }^{(6)}$.

\section{Procedimento e análise}

Se o serviço possuir, ou puder obter, o equipamento necessário para testar a blindagem da RF, os testes podem ser feitos em várias etapas durante a instalação. $\mathrm{O}$ equipamento deve incluir: a) um gerador capaz de produzir sinais na faixa apropriada de freqüência. Se o gerador não for capaz de produzir sinais de amplitude suficiente, considerando uma atenuação esperada de 100 dB, um amplificador será necessário; b) duas antenas calibradas para as devidas freqüências; c) um receptor, como um analisador de espectros, cobrindo toda a faixa de freqüências com sensibilidade adequada, de modo que a combinação de ganho e sensibilidade permitam medidas de atenuação dentro dos limites necessários.

$\mathrm{O}$ primeiro teste deve ser realizado na finalização da colocação da blindagem. Este teste é necessário para confirmar que a blindagem está de acordo com as necessidades de atenuação especificadas pelo fabricante do equipamento de IRM. Outro teste deve ser realizado após o fabricante instalar o magneto.

Testes adicionais podem ser feitos após a completa instalação do equipamento. Empresas independentes podem ser contratadas para realizar os testes, e os resultados devem ser repassados para o físico responsável na forma de um relatório ${ }^{(6,8)}$.

\section{Critério de aceitação}

Qualquer medida fora dos valores de atenuação especificados pelo fabricante deve ser comunicada ao responsável pela instalação da blindagem e ao responsável da instituição, para que sejam tomadas as medidas corretivas necessárias ${ }^{(\mathbf{6})}$.

\section{Teste 3 - Consumo do criogênio (taxa de evaporação)}

\section{Definição}

A taxa na qual o criogênio é consumido deve ser especificada pelo fabricante do equipamento de IRM na proposta de venda. Diferentes magnetos possuem taxas de consumo dos criogênios variáveis e o gasto com a reposição periódica pode representar um custo adicional bastante considerável, principalmente se a taxa de consumo estiver acima dos valores especificados pelo fabricante ${ }^{(6,8)}$.

\section{Procedimento e análise}

Determinar o consumo, em litros, do criogênio utilizado (nitrogênio líquido ou hélio) para um dado período de tempo, sendo que, quanto mais longo for o período de tempo, melhor. Alguns fabricantes medem o consumo em valores porcentuais (\%) da capacidade total do magneto, que devem ser convertidos para litros através de tabelas previamente fornecidas pelo próprio fabricante. A taxa de consumo calculada é obtida dividindo-se o total de litros utilizado pelo período de tempo em horas correspondente. Desta forma, o consumo ficará expresso em litros por hora.

\section{Critério de aceitação}

Se o valor estiver acima de $30 \%$ do valor especificado, deve-se entrar em contato imediatamente com o fabricante e verificar possíveis falhas no abastecimento de energia dos sistemas que controlam as bombas de circulação do "cold head" do magneto $^{(6,8)}$.

\section{Teste 4 - Homogeneidade do campo magnético principal $\left(B_{0}\right)$ \\ Definição}

A homogeneidade do campo magnético principal $\left(\mathrm{B}_{0}\right)$ se refere à uniformidade da intensidade do campo magnético principal em um determinado volume. Esta é usualmente especificada em partes por milhão da intensidade do campo magnético principal, em um dado diâmetro de volume esférico (dsv).

A homogeneidade é influenciada por vários fatores, incluindo inomogeneidades no campo $\mathrm{B}_{0}$ produzidas por imperfeições nas espiras das bobinas. Inomogeneidades podem contribuir para distorção geométrica das imagens, influenciando de forma negativa a uniformidade da imagem e comprometendo a razão sinal-ruído (RSR) em algumas seqüências rápidas ${ }^{(6,17)}$.

\section{Procedimento e análise}

Método 1 - Sonda de campo magnético. $\mathrm{O}$ engenheiro de instalação mede a homogeneidade do campo magnético com dados de uma sonda de RF, na qual pequenas amostras de água são precisamente posicionadas em vários pontos dentro do magneto e dados são coletados ${ }^{(6,17)}$.

Método 2 - Picos espectrais. Posicionar um dispositivo de teste esférico e uniforme no isocentro do magneto. O simulador esférico deve possuir diâmetro similar ao valor de diâmetro do volume sensível (dsv) especificado pelo fabricante. Produzir um espectro da amostra. Ter certeza de que a resolução em frequiência é muito menor que a largura do pico esperado. Medir a largura máxima a meia altura (FWHM) do pico espectral. Converter a FHWM de hertz $(\mathrm{Hz})$ para partes por milhão (ppm) da intensidade do campo magnético $\mathrm{B}_{0}$, usando a equação de Larmor modificada, para que o resultado apareça com a unidade ppm, como mostrado abaixo:

$$
\begin{array}{r}
\text { FWHM }(\mathrm{ppm})=\mathrm{FWHM}(\mathrm{Hz}) / \gamma \cdot \mathrm{B}_{0} \\
(\text { equação } 1)
\end{array}
$$

A FWHM (ppm) define a inomogeneidade sobre o volume do simulador utilizado.

Método 3 - Mapa de diferença de fase. Este teste oferece uma medida precisa da homogeneidade do $\mathrm{B}_{0}$ usando um dispositivo de teste uniforme. Entretanto, o teste necessita de certas habilitações do sistema (isto é, mostrar imagens de fase) que geralmente não estão disponíveis em todas as unidades de IRM para o operador.

Os efeitos de não-linearidade nos gradientes e inomogeneidade podem ser separados através do mapeamento da intensidade do campo $B_{0}$. Se o sistema de IRM 
pode realizar subtração e mostrar imagens de fase, uma medida pixel a pixel das inomogeneidades pode ser obtida.

O procedimento inclui posicionar o simulador no centro da bobina de corpo. $\mathrm{O}$ simulador deve incluir, no mínimo, um diâmetro circular de $10 \mathrm{~cm}$, ou $85 \%$ da área especificada na proposta de venda, qualquer que seja o maior. Empregar uma sequiência de pulso com gradiente arrasador. O uso de uma seqüência spin eco irá resultar em uma refasagem das diferenças de fase devido a inomogeneidades do campo magnético. Adquirir uma imagem usando um tempo de eco entre 30 e $40 \mathrm{~ms}\left(\mathrm{TE}_{1}\right)$ e mostrar esta imagem como um mapa de fase. Adquirir outra imagem usando um TE de alguns milissegundos maior $\left(\mathrm{TE}_{2}\right)$. Subtrair a segunda imagem da primeira e obter uma imagem na qual cada intensidade de pixel represente a diferença de fase entre duas aquisições, uma vez que o tempo T2 da solução foi sempre o mesmo.

A diferença $\left(\Delta \mathrm{B}_{0}\right)$ entre o campo magnético $\mathrm{B}_{0}$ em um dado voxel e o valor de referência no centro do campo de visão (CDV) é:

$$
\Delta \mathrm{B}_{0}=\delta \phi / \gamma\left(\mathrm{TE}_{1}-\mathrm{TE}_{2}\right), \quad \text { (equação 2) }
$$

onde: $\Delta \mathrm{B}_{0}$ é dado em mT; $\delta \phi$ é a diferença de fase expressa em radianos; $\gamma$ é a razão giromagnética para o hidrogênio (42,576 $\mathrm{Hz} / \mathrm{mT}$ ); TE é expresso em milissegundos. Este procedimento pode ser repetido para que sejam obtidos dados nos três principais planos (axial, sagital e coronal).

Determinar a maior diferença em qualquer plano entre os valores de $\mathrm{DB}_{0}$ dentro de regiões de interesse (ROI) circulares que possuam o valor especificado de diâmetro (dsv). Este valor dividido pelo $\mathrm{B}_{0}$ do magneto irá fornecer a homogeneidade (em ppm) para o dado diâmetro do volume esférico $(\mathrm{dsv})^{(6,17)}$.

\section{Critério de aceitação}

Valores típicos estão em torno de 2 ppm para um dsv de 30 a $40 \mathrm{~cm}$ em magnetos supercondutores. Imagens de fase podem mostrar deformações nas regiões onde a fase excede $\pm 180^{\circ}$ em relação ao valor de referência. Algoritmos antideformação podem ser empregados para eliminar este fator. A cooperação do engenheiro de serviço do fabricante pode ser necessária para que se obtenha acesso a rotinas de pósprocessamento no equipamento.

Uma falta de homogeneidade de campo magnético pode estar relacionada a pequenos objetos ferromagnéticos atraídos para o interior do magneto, como grampos de papel e moedas. Mudanças na localização de grandes objetos ferromagnéticos no ambiente em torno da sala de exames também podem causar inomogeneidades do campo magnético.

\section{Teste 5 - Exatidão da espessura de corte}

\section{Definição}

A espessura de corte é definida como a FWHM de um perfil de corte. O perfil de corte é definido como a resposta de um sistema de IRM a uma fonte puntiforme, à medida que esta se move através do plano de reconstrução deste ponto ${ }^{(4,6-8,17,18)}$.

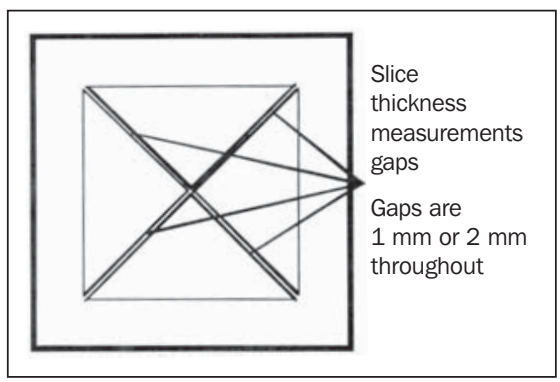

Figura 2. Esquema representativo das rampas cruzadas do dispositivo de teste 3DRAS.

\section{Procedimento e análise}

Os conjuntos de rampas cruzadas produtoras de sinal do dispositivo de teste 3DRAS (Figura 2) permitem que a espessura de corte possa ser medida mediante obtenção de imagens com uma típica aquisição multicortes com ponderação $\mathrm{T} 1^{(19)}$. Imagens em cortes axial, sagital e coronal devem ser obtidas de forma que cada uma delas corte as rampas cruzadas, como mostra a Figura 3.

$\mathrm{Na}$ imagem resultante (Figura 4), a intensidade de sinal é medida pixel a pixel, atravessando a rampa com uma linha de interesse (linhas A e B) orientada ortogonalmente à direção de largura da rampa. Um "software" de domínio público pode ser usado para realizar esta etapa ${ }^{(20)}$.

De posse dos dois perfis de intensidades de sinal da imagem das duas rampas, como mostra a Figura 5, é possível medir a FWHM. A FWHM das rampas opostas, orientadas a um ângulo de $45^{\circ}$ em relação ao plano de imagem, é igual à raiz quadrada do produto de $a$ e $b$, onde $a$ e $b$ são as FWHM das rampas 1 e $2^{(4,6-8,17-19)}$.

\section{Critério de aceitação}

A espessura de corte pode variar de acordo com a distância ao centro do magneto em aquisições multicortes. Assegurando uma precisão adequada na medida, o valor da espessura de corte deve coinci-

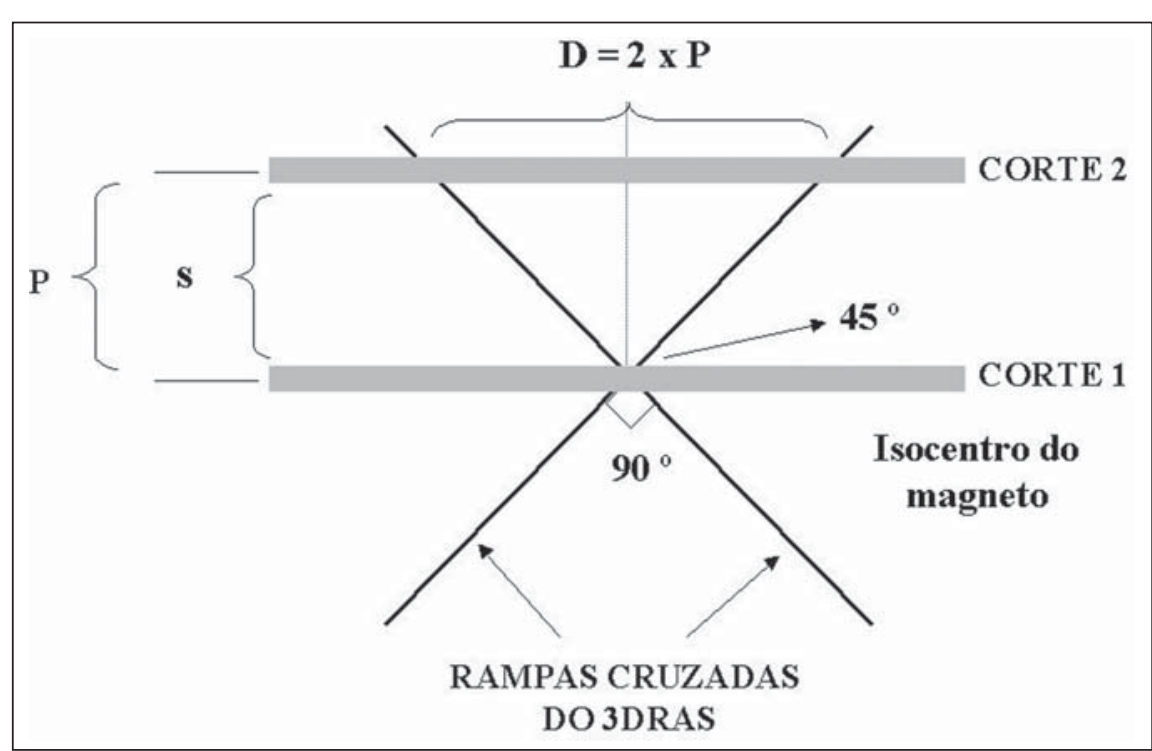

Figura 3. Esquema representativo de dois cortes posicionados de forma a interceptar as rampas cruzadas em $45^{\circ}$. P é a distância entre o centro de cada corte e S é o espaçamento entre cortes. D é a distância que será medida na imagem para que seja conhecido o espaçamento P. 


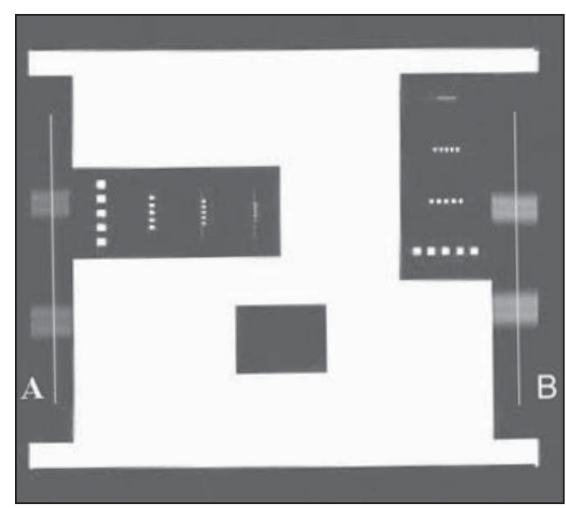

Figura 4. Imagem do dispositivo de teste 3DRAS mostrando o posicionamento das linhas A e B sobre as imagens em corte das rampas cruzadas que originarão os perfis de intensidade de sinal.

dir com o valor selecionado dentro de um intervalo de $\pm 1 \mathrm{~mm}$, para espessuras de corte selecionadas com mais de $5 \mathrm{~mm}$.

Teste 6 - Exatidão do posicionamento e do espaçamento de corte

\section{Definição}

A posição de corte é a localização absoluta do ponto médio da FWHM de um perfil de corte. A separação ou espaçamento entre cortes é a distância entre quaisquer duas posições de corte. As localizações de corte são indicadas por dispositivos de posicionamento externos ou pelo espaçamento entre os cortes selecionados ${ }^{(\mathbf{4 , 6 - 8 , 1 7 - 1 9})}$.

\section{Procedimento e análise}

Uma imagem de cada plano do dispositivo de teste 3DRAS deve ser utilizada. A imagem central de cada plano não deve ser utilizada para as medidas mas sim para verificar se o dispositivo se encontra corretamente posicionado no centro do magneto (isocentro) ${ }^{(\mathbf{1 9})}$. O ponto médio da FWHM do perfil de corte da imagem de interesse deve ser determinado. A distância do ponto médio do primeiro perfil até o ponto médio do segundo perfil (D), dividido por dois, representa a localização, ou posição do corte, uma vez que as rampas possuem inclinação de $45^{\circ}$ com o plano de imagem. É importante notar a necessidade do corte central cruzar exatamente o ponto de interseção das duas rampas. A distância entre o final do perfil da rampa 1 e o início do perfil de corte da rampa 2

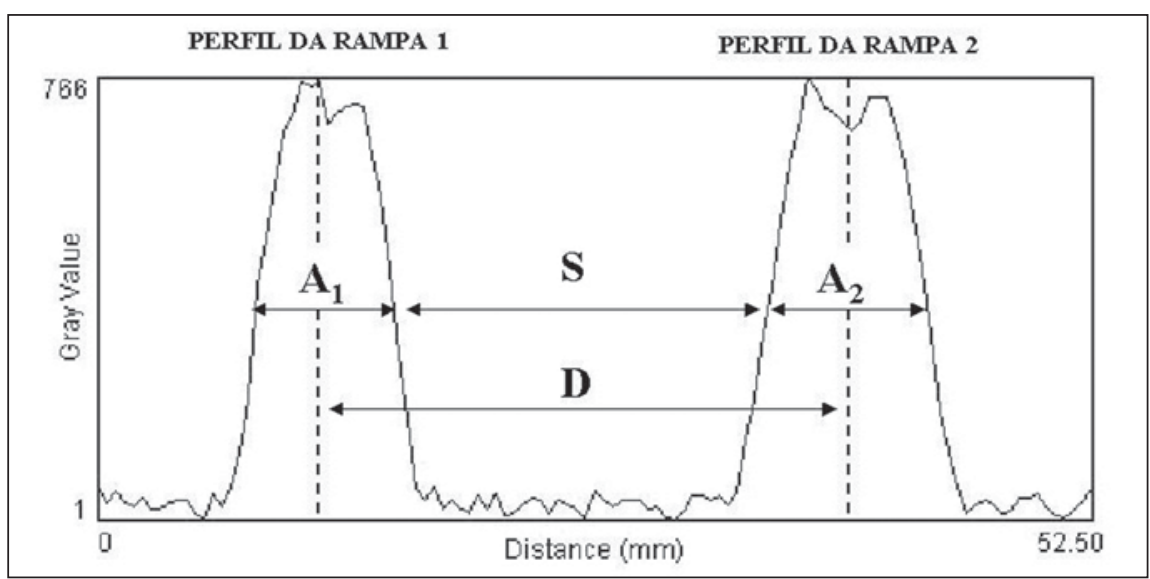

Figura 5. Perfil da intensidade de sinal originado da colocação de uma linha cruzando as rampas. $A_{1}$ é a largura a meia altura do perfil $1, A_{2}$ é a largura a meia altura do perfil $2, S$ é a distância entre $A_{1}$ e $A_{2}$ e D é a distância até o centro de cada corte. Estes valores permitirão a obtenção de dados sobre a espessura, espaçamento e posição do corte.

(S), na altura da FWHM, representa o es-

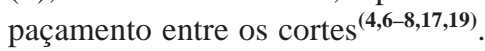

\section{Critério de aceitação}

O cálculo do valor obtido deve coincidir com o valor da posição em $\pm 2 \mathrm{~mm}$. Para o valor da separação, a diferença deve ser menor que $20 \%$ do espaçamento entre os cortes ou $\pm 1 \mathrm{~mm}$, o que for maior ${ }^{(4,7,8,17,18)}$.

\section{Teste 7 - Resolução espacial de alto contraste}

\section{Definição}

A resolução espacial de alto contraste é a medida da capacidade de um sistema de imagens em mostrar a separação entre objetos quando não existe uma contribuição significativa do ruído ${ }^{(7)}$.

Uma falha neste teste significa que, para um dado campo de visão (CDV) e uma dada matriz de aquisição, o equipamento não está diferenciando pequenos detalhes como deveria. Como conseqüência, pequenas lesões ou a definição das bordas de pequenas estruturas não aparecerão nas imagens produzidas por este aparelho.

\section{Procedimento e análise}

Uma imagem de cada plano do dispositivo de teste 3DRAS deve ser utilizada. A imagem será analisada visualmente para determinar o menor conjunto de furos mais resolvido, podendo utilizar a ferramenta de magnificação, conforme mostra a Figura 6. Para considerar que um conjunto está resolvido, ou seja, diferenciado dos demais, todos os furos e espaços devem estar aparecendo separados. O nível e a largura da janela devem ser ajustados para proporcionar uma ótima visualização. O 3DRAS possui dois conjuntos para cada plano, orientados ortogonalmente entre $\mathrm{si}^{(\mathbf{1 9})}$

\section{Critério de aceitação}

A resolução espacial de alto contraste deve permanecer constante para repetidas medidas feitas sob as mesmas condições de aquisição e deve ser igual ao tamanho do pixel. Por exemplo, para um campo de visão de $25 \mathrm{~cm}$ com uma matriz de $256 \times$ 256, a resolução deve ser de $1 \mathrm{~mm}^{(7,17,18)}$.

\section{Teste 8 - Uniformidade da imagem}

\section{Definição}

A uniformidade da imagem reflete a habilidade de um equipamento de IRM em representar regiões similares com a mesma intensidade em um volume homogêneo. Uma não-uniformidade pode ser resultado de inomogeneidades do campo magnético ou RF, correntes de "eddy", penetração e geometria da bobina de RF, calibração inadequada dos gradientes de pulso e processamento da imagem ${ }^{(6-8,17,18)}$.

\section{Procedimento e análise}

Uma imagem de cada plano do dispositivo de teste UAL deve ser utilizada. A imagem de cada plano deve ser da região preenchida somente pelo líquido produtor de sinal $\left(\mathrm{CuSO}_{4}\right)$. Uma ROI deve estar centrada no volume produtor de sinal, de 


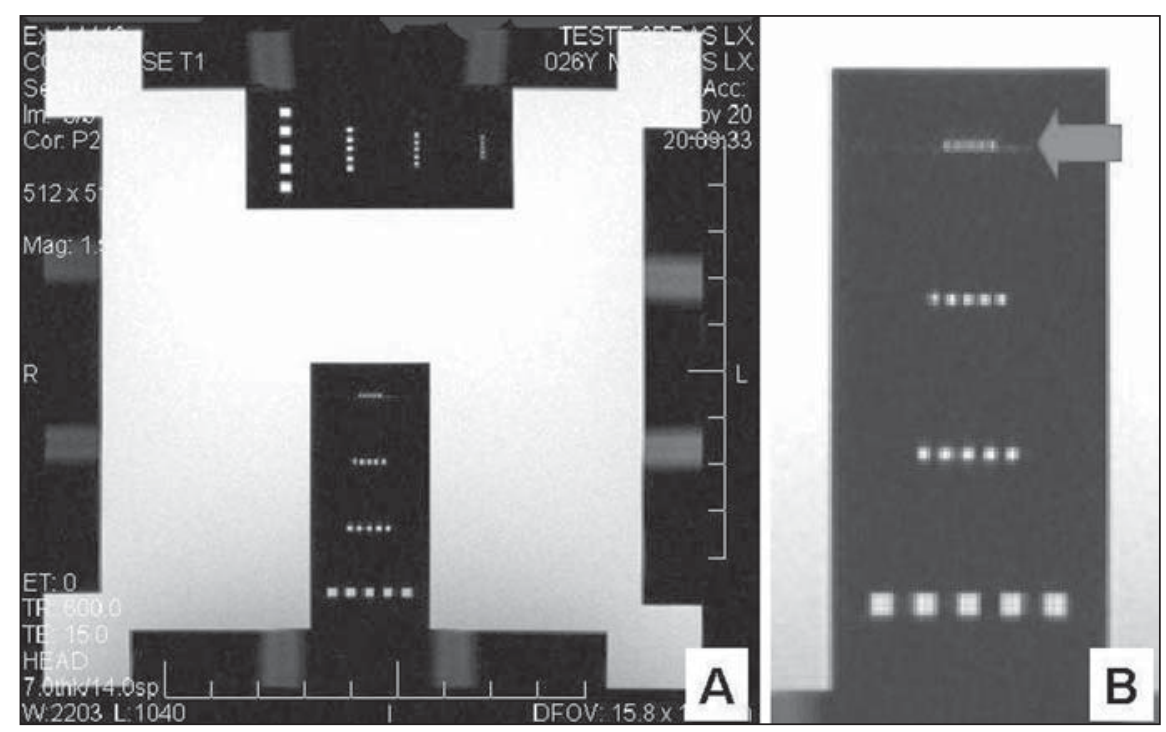

Figura 6. A: Imagem do dispositivo de teste 3DRAS, na posição em que serão avaliados os conjuntos de furos para medida da resolução espacial. B: Imagem ampliada de um dos conjuntos, onde a seta indica o menor conjunto com furos resolvidos.

forma que encubra $75 \%$ da imagem, excluindo, porém, regiões próximas às bordas que possam conter artefatos. Determinar o mínimo $\left(S_{\text {mín }}\right)$ e o máximo $\left(S_{\text {máx }}\right)$ valor de intensidade de pixel dentro da ROI. Calcular o porcentual de uniformidade integral (U) através da seguinte fórmula para cada plano de imagem ${ }^{(6-8,17,18)}$ :

$$
U=\left(1-\left(\frac{S_{\text {máx }}-S_{\text {mín }}}{S_{\text {máx }}+S_{\text {mín }}}\right)\right) \times 100 \%
$$

(equação 3)

\section{Critério de aceitação}

Para um campo de visão de $20 \mathrm{~cm}$ ou menos, a uniformidade integral deve ser de $80 \%$ ou mais. É conveniente lembrar que, para grandes campos de visão, a uniformidade pode sofrer deterioração devido aos efeitos de atenuação da $\mathrm{RF}^{(\mathbf{6 - 8 , 1 7 , 1 8})}$. Esta metodologia para teste de uniformidade não deve ser utilizada para bobinas de superfície $^{(17)}$.

\section{Teste 9 - Linearidade espacial ou distorção geométrica}

\section{Definição}

Linearidade espacial é o termo utilizado para descrever o grau de distorção geométrica presente nas imagens produzidas por um sistema de IRM. A distorção geométrica pode se referir tanto ao deslocamento de pontos dentro de uma imagem em rela- ção à sua localização real como à calibração da escala de distância entre quaisquer pontos dentro da imagem ${ }^{(6-8,10,17-19)}$.

\section{Procedimento e análise}

Uma imagem de cada plano do dispositivo de teste UAL deve ser utilizada. A imagem de cada plano escolhida deve ser ortogonal à grade de acrílico. As variações geométricas são melhor observadas quando é utilizado o maior campo de visão possível, devendo o dispositivo de teste UAL ocupar pelo menos $60 \%$ deste campo de visão ${ }^{(\mathbf{1 9})}$. Uma consideração a ser feita é a de utilizar uma aquisição com o maior tamanho de matriz (por exemplo, $512 \times 512$ ) para maximizar a resolução espacial $^{(\mathbf{8 , 1 9})}$. As medidas devem ser feitas recomendável que as distâncias sejam sempre maiores que dez pixels. Para cada medida em cada plano de imagem deve ser calculado o porcentual de distorção geométrica (DG) através da fórmula ${ }^{(6-8,10,17,19)}$ :

$$
D G=\frac{d_{V}-d_{M}}{d_{V}} \times 100 \% \quad(\text { equação } 4)
$$

onde: $\mathrm{d}_{\mathrm{V}}$ é a distância verdadeira ou real; $\mathrm{d}_{\mathrm{M}}$ é a distância medida.

\section{Critério de aceitação}

O porcentual de distorção da linearidade é aceitável se for inferior a 5\%(6-8,10,19). entre quaisquer dois pontos da grade. É

\section{Teste 10 - Análise de artefatos na imagem}

\section{Definição}

Erros relacionados à fase são definidos em termos de intensidade de sinal na imagem (tanto aumentada como diminuída) em localizações específicas. Geralmente, estes artefatos são caracterizados por áreas de intensidade de sinal aumentado onde não deveria haver tal sinal. Assim sendo, foram apelidados de "fantasmas"(19).

Uma série de erros pode ser detectada através das imagens obtidas:

a) Erros de codificação de fase: fantasmas por codificação de fase irão aparecer como múltiplas imagens (possivelmente espalhadas em uma coluna) originárias de um objeto produtor de sinal real na direção do gradiente de codificação de fase da imagem. A presença destas imagens fantasmas características irá geralmente identificar os dois eixos ${ }^{(19)}$.

b) Erros de DC-“offset": aparecem geralmente como pixels brilhantes (ou algumas vezes escuros) no centro da matriz da imagem $^{(19)}$.

c) Erros do receptor de quadratura: aparecem do lado oposto e abaixo em relação ao objeto produtor de sinal. Se o objeto estiver no lado esquerdo e acima, a imagem fantasma irá aparecer abaixo e à esquerda ${ }^{(19)}$.

d) Erros do transmissor de quadratura: a imagem fantasma aparece num corte localizado na direção oposta ao deslocamento do dispositivo de teste do centro do magneto e a uma distância igual a este deslocamento. A imagem fantasma e o objeto produtor de sinal estarão localizados na mesma posição relativa nas imagens. Por exemplo, se o objeto real está localizado no canto superior esquerdo a uma distância de $+5,0 \mathrm{~cm}$ do isocentro, a imagem fantasma estará no canto superior esquerdo de uma imagem a $-5,0 \mathrm{~cm}^{\mathbf{( 1 9 )}}$.

\section{Procedimento e análise}

Uma imagem de cada plano do dispositivo de teste UAL deve ser utilizada. Estas devem mostrar a imagem do pequeno cilindro produtor de sinal preso à parede do dispositivo de teste.

Para avaliar o erro de codificação de fase, DC-“offset" e transmissor de quadra- 
tura, o dispositivo de teste UAL deve ser posicionado no isocentro do equipamento. Para a verificação do erro do receptor de quadratura, o cilindro produtor de sinal preso à parede do dispositivo de teste UAL é que deve estar no isocentro do equipamento.

A avaliação das imagens geradas em cada plano é feita da seguinte forma:

a) Erros de codificação de fase: deve ser posicionada uma ROI sobre a imagem verdadeira do cilindro produtor de sinal e uma ROI sobre a imagem fantasma mais brilhante. O valor de cada ROI deve ser registrado para que a magnitude do erro (E) seja expressa pelo valor da ROI da imagem fantasma $(\mathrm{G})$ como um porcentual do valor da ROI da imagem real (T), da seguinte forma ${ }^{(19)}$ :

$$
E=((T-G) / T) \times 100 \% \quad(\text { equação 5) }
$$

b) erros de DC-“offset": a presença das imagens fantasmas deve ser registrada após uma inspeção visual ${ }^{(\mathbf{1 9 )}}$;

c) erros do receptor de quadratura: os valores da ROI, tanto da imagem verdadeira como da imagem fantasma, devem ser registrados. O erro do receptor de quadratura (E) é quantificado usando-se a equação $5^{(19)}$;

d) erro do transmissor de quadratura: os valores da ROI, tanto da imagem verdadeira como da imagem fantasma, devem ser registrados. O erro do transmissor de quadratura (E) é quantificado usando-se a equação $5^{(19)}$.

\section{Critério de aceitação}

$\mathrm{O}$ valor de $\mathrm{E}$ deve ser inferior a $5 \%$, sendo que o erro de DC-“offset" não deve ser encontrado em sistemas de IRM que estejam funcionando adequadamente ${ }^{(\mathbf{1 9})}$.

\section{Teste 11 - Estabilidade do campo magnético ou freqüência central}

\section{Definição}

A frequiência de ressonância é definida como a freqüência (w) que coincide com o valor do campo magnético principal $\left(\mathrm{B}_{0}\right)$ de acordo com a equação de Larmor. Antes da aplicação de qualquer protocolo de imagem, é essencial saber se o equipamento de IRM está "em ressonância". Muitos fabricantes dos equipamentos fornecem protocolos específicos para ajustes da fre- qüência de ressonância. Atualmente, a maioria dos equipamentos realiza o ajuste da frequiência central de forma completamente automática antes da aquisição das imagens ${ }^{(17)}$.

\section{Procedimento e análise}

É utilizado dispositivo de teste de RSR de crânio (GE Medical Systems). O dispositivo é posicionado no centro do magneto dentro da bobina de crânio. Durante os ajustes automáticos de pré-aquisição, o equipamento fornece o valor da freqüência central $\mathrm{f}_{0}$ e os valores de ganho de transmissão e ajuste dos receptores de RF. Todos estes valores devem ser anotados.

\section{Critério de aceitação}

Valores típicos de frequiência de ressonância não devem sofrer desvio superior a 1,5 ppm num período de 24 horas. Se o valor exceder a $1,5 \mathrm{ppm}$, deve-se repetir o teste. Se o resultado voltar a exceder 1,5 ppm, o fabricante ou o responsável pela manutenção devem ser notificados ${ }^{(17)}$.

\section{Teste 12 - Ganho de transmissão ou atenuação}

\section{Definição}

Mudanças no ganho de transmissão (GT) estão diretamente relacionadas a mudanças na RSR. Uma medida do ganho de transmissão é útil como primeira avaliação do sistema e não necessita de tempo extra, uma vez que o valor é medido automaticamente durante a pré-aquisição ${ }^{(17)}$.

\section{Procedimento e análise}

Durante a aquisição que avalia o valor da freqüência central, usando o dispositivo de teste para RSR do crânio, o valor do GT e os valores dos receptores (R1 e R2) são $\operatorname{anotados}^{(17)}$.

\section{Critério de aceitação}

Alterações superiores a $30 \%$ do valor esperado do GT devem ser comunicadas ao fabricante. É importante relacionar o GT com aumento de ruído nas imagens e diminuição da $\operatorname{RSR}^{(17)}$.

\section{Teste 13 - Checagem das bobinas de RF Definição}

A fabricação e o desenho das bobinas de RF sempre representam uma negocia- ção entre maximizar a uniformidade da imagem e aumentar a RSR. As bobinas que são desenvolvidas para coletar sinal de partes do corpo colocadas dentro do seu volume (bobinas de crânio e corpo, por exemplo) irão produzir uma excitação e uma recepção do sinal de RM bastante uniforme. Estas bobinas são chamadas de bobinas de volume ${ }^{(17)}$.

Outros exames são realizados usando as chamadas bobinas de superfície, que aumentam a RSR através da combinação das seguintes estratégias:

a) Posicionar a bobina o mais próximo possível do volume de interesse;

b) excluir o sinal e o ruído de regiões que não estão sob investigação;

c) maximizar o sinal do volume de interesse na região sensível da bobina.

Tipicamente, estas bobinas de superfície geram imagem com baixa uniformidade. Contudo, se o radiologista está consciente destas características, a percepção da imagem pode ser condicionada no momento da interpretação. Recentemente, uma nova classe de bobinas de RF, chamadas de conjunto de fase, tem sido empregada para melhorar a uniformidade da imagem.

Para as bobinas de volume, três parâmetros serão avaliados: uniformidade da imagem (U), RSR e o porcentual de artefato fantasma (PAF). Juntos, estes três parâmetros podem ser usados para caracterizar o desempenho de uma bobina e rastrear alterações ao longo dos anos.

Para as bobinas de superfície, somente o valor máximo da RSR será medido. Os outros parâmetros serão verificados visualmente, para observar se não existem artefatos de imagens fantasmas ou grande redução de uniformidade dentro do volume de interesse.

\section{Procedimento e análise}

A aquisição das imagens necessárias para avaliar os parâmetros descritos acima, para cada tipo de bobina, segue o mesmo procedimento:

a) Posicionar a bobina no magneto e dentro de seu volume sensível, colocar o dispositivo de teste apropriado. O dispositivo deve abranger pelo menos $80 \%$ do campo de visão e o material de preenchimento deve estar de acordo com as especificações; 
b) utilizar sequiência de pulso e parâmetros técnicos típicos para o tipo de exame utilizado com a dada bobina;

c) duas aquisiçõos consecutivas (duas imagens) da região central dos dispositivos de teste em cada bobina devem ser obtidas. Observar o plano de corte (axial, sagital ou coronal).

\section{a) Teste de uniformidade das bobinas de volume}

Definição - Como já definido, a uniformidade da imagem reflete a capacidade de um equipamento de IRM em representar regiões similares com a mesma intensidade em um volume homogêneo.

Procedimento e análise - Após escolher uma das duas imagens obtidas, uma ROI deve ser centrada no volume produtor de sinal de modo que encubra $75 \%$ da imagem, porém exclua regiões próximas às bordas. Determinar o mínimo $\left(S_{\text {mín }}\right)$ e o máximo $\left(S_{\text {máx }}\right)$ valor de pixel dentro da ROI. Calcular o porcentual de uniformidade integral (U) pela equação $3^{(\mathbf{1 7})}$.

Critério de aceitação - Para um campo de visão de $20 \mathrm{~cm}$ ou menos, a uniformidade integral deve ser de $80 \%$ ou mais. É conveniente lembrar que, para grandes campos de visão, a uniformidade pode sofrer deterioração devida a efeitos de atenuação da $\mathrm{RF}^{(\mathbf{1 7})}$.

\section{b) Razão sinal-ruído}

Definição - A imagem da RSR é um parâmetro relacionado à utilidade clínica de IRM e, também, é uma medida sensível do desempenho do equipamento.

O sinal é definido como o valor médio do pixel dentro de uma ROI. O ruído é definido como variações randômicas na intensidade de sinal desta mesma ROI. Imagens que contenham artefatos óbvios não devem ser utilizadas para realizar o teste de sinal-ruído ${ }^{(\mathbf{1 7})}$.

Procedimento e análise - Uma ROI deve ser posicionada na imagem 1 e na imagem 2. Cada uma das regiões deve incluir $75 \%$ da imagem. A média entre os dois valores de intensidade de sinal dentro das regiões deve ser calculada. Este valor é denominado de sinal (S).

Através de um programa instalado nos dois equipamentos de IRM, uma terceira imagem deve ser gerada da subtração da imagem 1 pela imagem 2. Uma nova ROI que também inclua $75 \%$ da imagem 3 deve ser posicionada. $\mathrm{O}$ desvio-padrão (DP) medido e fornecido pelo equipamento é chamado de ruído $(\mathrm{R})$.

A RSR pode ser calculada através da seguinte equação:

$$
R S R=\sqrt{2} \times \frac{S}{R}
$$

O fator $\sqrt{2}$ é necessário, pois o ruído é derivado de uma imagem subtraída e não de uma imagem original ${ }^{(\mathbf{9})}$.

Critério de aceitação - As medidas da RSR obtidas com o dispositivo de teste fornecido pelo fabricante podem ser comparadas com valores padrões do próprio fabricante, se estes estiverem disponíveis.

\section{c) Porcentual de artefato fantasma}

Definição - Assim como para a análise de artefatos nas imagens produzidas pelo dispositivo de teste UAL, para cada bobina verificada devem ser procurados artefatos tipo fantasma. As imagens de magnitude reconstruídas são usadas como imagens clínicas de RM por serem relativamente imunes a erros de fase no sinal de RM. Quando estes erros se tornam maiores, eles podem resultar em imagens fantasmas mesmo nas imagens de magnitude. Artefatos tipo fantasma também podem ser gerados por movimento ou vibração do dispositivo de teste durante a aquisição. É preciso ter total atenção com a estabilidade do dispositivo de teste dentro da bobina.

Inconsistências periódicas da amplitude podem resultar em tipos de artefatos facilmente identificáveis. $\mathrm{O}$ artefato fantasma tipo "metade do CDV" ou "fantasma N/2" é bastante comum em imagens eco-planares (EPI), e é também muito difícil de ser eliminado por completo.

Procedimento e análise - O PAF é obtido através dos seguintes passos:

1) Criar uma ROI que cubra $75 \%$ da área da imagem do dispositivo de teste e registrar o valor médio das intensidades de pixel como sinal médio (SM);

2) colocar uma ROI numa posição fora dos limites da imagem do dispositivo de teste na direção de codificação de freqüência para não incluir possíveis artefatos tipo fantasma. Uma boa localização é no canto superior direito da imagem;
3) determinar o sinal de fundo (SFU) como o valor médio das intensidades de pixel contidas na ROI;

4) mover a ROI para uma posição também fora da imagem do dispositivo de teste, porém na direção de fase. Este será chamado de sinal fantasma (SFA). A Figura 7 fornece um exemplo de posicionamento das ROI;

5) o PAF é calculado usando-se a seguinte equação:

$$
\begin{aligned}
P A F=\frac{(S F A-S F U)}{(2 \times S M)} \times 100 \% \\
(\text { equação 7) }
\end{aligned}
$$

Critério de aceitação - $\mathrm{O}$ valor do $\mathrm{PAF}$ deve ser inferior a $5 \%$, sendo que o erro de DC-“offset" não deve ser registrado em sistemas de IRM que estejam funcionando adequadamente $^{(\mathbf{1 7 )}}$.

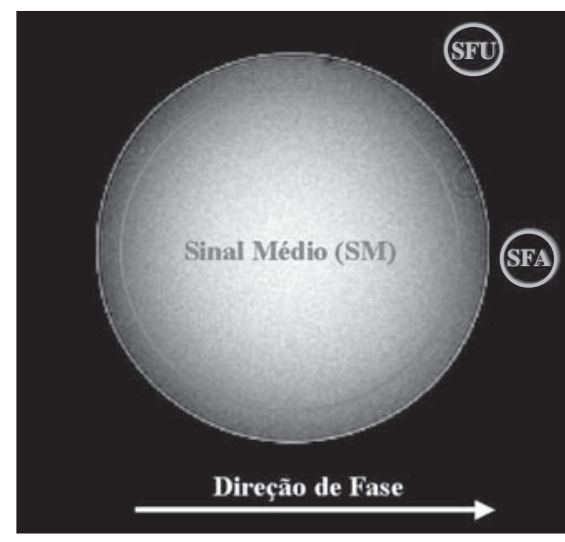

Figura 7. Exemplo de posicionamento das regiões de interesse para avaliação do porcentual de artefato fantasma.

\section{Teste 14 - Interferência de RF entre os cortes}

\section{Definição}

Em aquisições multicortes, a excitação dos prótons de hidrogênio e a aquisição dos dados a partir de múltiplos cortes são efetuadas dentro de um tempo de repetição (TR). Se existir pequeno espaço entre os cortes, há risco muito grande de ocorrer interferência entre eles ("cross talk"), uma vez que os perfis de excitação de um corte não são perfeitos. $\mathrm{O}$ que podemos esperar é uma redução na RSR, pois os prótons de um corte podem ser parcialmente excitados por um pulso de RF criado para excitar os prótons de outro corte ${ }^{(\mathbf{1 7 )}}$. 
Os diferentes fabricantes de equipamentos de IRM têm desenvolvido uma variedade de soluções para este tipo de problema. Alguns sistemas de IRM permitem adquirir imagens sem espaço entre cortes. Outros permitem um espaço mínimo de $10 \%$ da espessura de corte. Por outro lado, muitos sistemas automaticamente adquirem os cortes numa determinada ordem que maximize a distância entre os cortes excitados sucessivamente. $\mathrm{O}$ método descrito abaixo é uma adaptação de Vlaardingenbroek e den Boer. ${ }^{(21)}$.

\section{Procedimento e análise}

Posicionar o dispositivo de teste de RSR de crânio na bobina de crânio, de forma que o centro do dispositivo coincida com o zero (isocentro) do equipamento. Obter as imagens com protocolo de aquisição que possua quatro diferentes séries, em que em cada série um espaço entre os cortes é utilizado (por exemplo, 0,0 mm, 0,5 mm, 1,0 mm e 5,0 mm $)^{(\mathbf{1 7})}$.

Visualizar a imagem central de cada série e posicionar uma ROI que cubra $80 \%$ da área da imagem. Registrar o valor médio das intensidades de pixel como SM.

Colocar uma ROI numa posição fora dos limites da imagem do dispositivo de teste na direção de codificação de freqüência, para não incluir possíveis artefatos tipo fantasma. Uma boa localização é no canto superior direito da imagem.

Determinar o DP do SFU como o valor médio das intensidades de pixel contidas na ROI. Este valor é chamado de ruído. Dividir o SM pelo DP e obter o valor da RSR. Criar um gráfico que relacione o porcentual de espaço entre corte (eixo x) com o valor RSR (eixo y). O valor da RSR deve ser normalizado para o valor da RSR obtida com porcentual de espaçamento de $100 \%$ da espessura de corte (i.é, 5,0 mm).

\section{Critério de aceitação}

Efeitos de interferência entre cortes ("cross talk") podem reduzir a RSR por mais de $20 \%$ quando comparamos o valor com $100 \%$ de espaçamento e $0 \%$ de espaçamento. Grandes reduções da RSR para aquisições com pequeno espaçamento podem, em cortes contíguos, causar degradação da imagem. Se houver uma redução maior que $20 \%$, deve-se entrar em conta- to com o fabricante para determinar a extensão do problema e explorar possíveis soluções ${ }^{(17,21)}$.

\section{Teste 15 - Verificação da espectroscopia}

\section{Definição}

A avaliação da espectroscopia tem a finalidade de verificar se as aquisições típicas para voxel único ("press" e "steam") estão de acordo com as recomendações do fabricante.

\section{Procedimento e análise}

O dispositivo de teste Braino (Figura 8), que simula os picos espectrais referentes a alguns metabólitos encontrados no tecido cerebral de um paciente normal, deve ser posicionado na bobina de crânio. A temperatura em que se encontra o dispositivo deve ser anotada e o valor deve ser fornecido ao equipamento de IRM para que este faça ajustes na escala de ppm do espectro. Os valores obtidos através da quantificação automática devem ser anotados para cada tipo de aquisição ("press" e "steam") e comparados, caso o fabricante possua valores de referência.

\section{Critérios de aceitação}

Devem ser observados todos os metabólitos especificados pelo dispositivo, e caso existam valores de referência, estes devem se encontrar dentro de uma faixa de aceitação de $30 \%$.

Alterações visuais na posição aparente dos picos e na sua largura devem ser registradas e comunicadas ao fabricante, para que o procedimento de calibração da espectroscopia seja realizado.

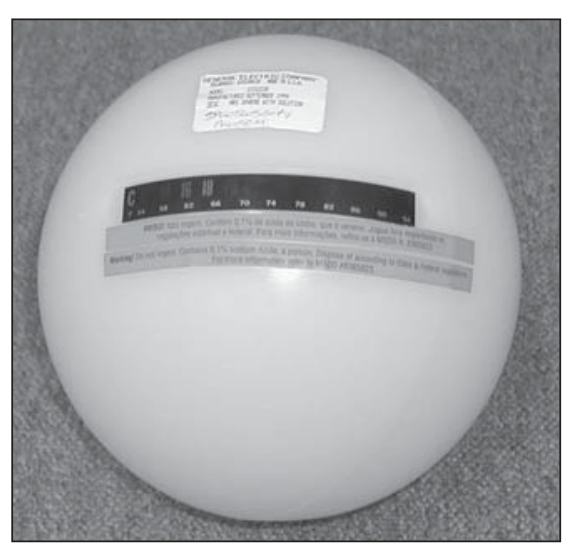

Figura 8. Foto do dispositivo de teste Braino.

\section{DISCUSSÃO}

A observação física dos equipamentos é simples de ser executada e mostra a conformidade na entrega dos itens relacionados na proposta de venda, assim como questões relacionadas à segurança do ambiente de RM.

Segundo Andrews ${ }^{(2)}$, manter registros diários do nível de hélio é uma boa forma de confirmar que o magneto está dentro das especificações. Não-conformidades no consumo de hélio podem indicar problemas na fabricação e instalação do magneto, assim como defeitos relacionados ao fornecimento de energia elétrica para o compressor de água fria, que obrigatoriamente deve permanecer ligado.

A exatidão da espessura, da posição e do espaçamento de corte chama a atenção para o cuidado que devemos ter em trabalhos que envolvem a medida de distâncias e o uso das imagens de RM para planejamento cirúrgico.

A freqüência central e o ganho de transmissão avaliados durante a verificação da bobina de crânio são úteis para fornecer, de forma rápida e prática, parâmetros de normalidade dos transmissores de RF e da estabilidade do campo magnético.

A checagem das bobinas de RF permite verificar se os equipamentos respondem da mesma forma a uma mesma bobina. Muitas vezes, um simples teste com uma bobina de volume (por exemplo, bobina de crânio) pode não detectar falhas graves do equipamento com uma ou mais bobinas de superfície. E uma bobina pode estar funcionando bem em um equipamento e não estar no outro.

A interferência de RF entre cortes não consta como teste sugerido pela maioria dos autores consultados, mas foi incluído neste trabalho por ser de execução simples, de grande aplicabilidade e constar como um dos testes sugeridos pelo $\mathrm{ACR}^{(\mathbf{1 7})}$.

\section{CONCLUSÃO}

Através dos testes de aceitação descritos é possível verificar características técnicas que constam nas propostas de venda dos fabricantes, assim como estabelecer valores de referência para serem utilizados em testes de constância. 
Testes simples, como a observação física, podem resultar em uma grande ferramenta para verificar a conformidade na entrega dos acessórios adquiridos e na segurança que não só o equipamento, mas o ambiente da RM, oferece aos pacientes e funcionários.

Testes de aceitação e de constância devem ser realizados para garantir a qualidade da imagem e assegurar que artefatos ainda não visíveis nas imagens clínicas não venham a prejudicar o diagnóstico médico.

Em muitos equipamentos, os programas de garantia da qualidade em IRM são realizados tendo como base, principalmente, as recomendações dos fabricantes. É possível, com este trabalho, verificar, através de um conjunto mais amplo de testes, o desempenho inicial e periódico de equipamentos de IRM.

\section{Agradecimentos}

Agradecemos a todos os médicos radiologistas, físicos, técnicos e demais funcionários do setor de Ressonância Magnética do Hospital Moinhos de Vento de Porto Alegre, em especial aos doutores Paulo Cesar Sanvitto e Flávio Franciosi Aesse.

\section{REFERÊNCIAS}

1. Instituto Brasileiro de Geografia e Estatística. Estatísticas da saúde: assistência médico-sanitária. Rio de Janeiro, RJ: Gráfica Digital, 1999.
2. Andrews DE. Magnet homogeneity and testing of MRI magnets. In: Dixon RD, ed. MRI: acceptance testing and quality control. Proceedings of AAPM Symposium. North Carolina: Medical Physics Publishing, 1988.

3. Bronskill MJ. MRI acceptance testing by the physicist. In: Dixon RD, ed. MRI: acceptance testing and quality control. Proceedings of AAPM Symposium. North Carolina: Medical Physics Publishing, 1988.

4. Dixon RD. MRI: acceptance testing and quality control. Proceedings of AAPM Symposium. North Carolina: Medical Physics Publishing, 1988.

5. Gray JE, Morin RL. MRI acceptance testing and quality control: physicists' procedures. In: Dixon RD, ed. MRI: acceptance testing and quality control. Proceedings of AAPM Symposium. North Carolina: Medical Physics Publishing, 1988.

6. Och JG, Clarke GD, Sobol WT, Rosen CW, Mun SK. Acceptance testing of magnetic resonance imaging systems: report of AAPM Nuclear Magnetic Resonance Task Group No. 6. Med Phys 1992;19: 217-29.

7. Price RR, Axel L, Morgan T, et al. Quality assurance methods and phantoms for magnetic resonance imaging: report of AAPM Nuclear Magnetic Resonance Task Group No. 1. Med Phys 1990;17:28795.

8. Wood ML, Price RR. Quality control programs for MRI. In: The Physics of MRI: 1992 AAPM Summer School Proceedings. American Institute of Physics. Medical Physics Monograph No. 21, 1993; 53:718-53.

9. National Electrical Manufacturers Association. MS1-94. Determination of signal to noise ratio (SNR) in diagnostic magnetic resonance imaging. Rosslyn, VA: NEMA, 1995.

10. National Electrical Manufacturers Association. MS2-89. Determination of two-dimensional geometric distortion in diagnostic magnetic resonance images. Rosslyn, VA: NEMA, 1989.

11. National Electrical Manufacturers Association.
MS3-89. Determination of image uniformity in diagnostic magnetic resonance images. Rosslyn, VA: NEMA, 1989.

12. National Electrical Manufacturers Association. MS4-89 (Revision 1998). Acoustic noise measurement procedure for diagnostic magnetic resonance imaging devices. Rosslyn, VA: NEMA, 1998.

13. National Electrical Manufacturers Association. MS5-91. Determination of slice thickness in diagnostic magnetic resonance imaging. Rosslyn, VA: NEMA, 1991.

14. National Electrical Manufacturers Association. MS6-91. Characterization of special purpose coils for diagnostic magnetic resonance images. Rosslyn, VA: NEMA, 1991.

15. National Electrical Manufacturers Association. MS7-93. (Revision 1998). Measurement procedure for time-varying gradient fields $(\mathrm{dB} / \mathrm{dt})$ for magnetic resonance imaging systems. Rosslyn, VA: NEMA, 1998.

16. National Electrical Manufacturers Association. MS8-93. Characterization of the specific absorption rate for magnetic resonance imaging systems. Rosslyn, VA: NEMA, 1993.

17. American College of Radiology. Magnetic resonance imaging quality control manual. ACR. Committee on Quality Assurance in MRI, 2001.

18. Lerski R, De Wilde J, Boyce D, Ridgeway J. Quality control in magnetic resonance imaging. IPEM Report No. 80. York, England: Institute of Physics and Engineering in Medicine, 1998.

19. Nuclear Associates. AAPM MRI phantoms. 3D resolution and slice (DRAS) phantom. Uniformity and linearity (UAL) phantom. Carle Place: Victoreen, 2001.

20. Rasband W. ImageJ version 1.24t. National Institutes of Health, USA. Disponível em: URL:http:// rsb.info.nih.gov/ij/. (citado 2001 out. 22).

21. Vlaardingerbroek MT, den Boer JA. Magnetic resonance imaging. 2nd ed. Berlim: Springer-Verlag, 1999. 